\title{
Pilot study of neoadjuvant chemotherapy with gemcitabine and oral S-1 for resectable pancreatic cancer
}

\author{
HIDEHIRO TAJIMA $^{1}$, TETSUO OHTA ${ }^{1}$, HIROHISA KITAGAWA ${ }^{1}$, KOICHI OKAMOTO ${ }^{1}$, SEISHO SAKAI ${ }^{1}$, \\ ISAMU MAKINO $^{1}$, JUN KINOSHITA ${ }^{1}$, HIROYUKI FURUKAWA ${ }^{1}$, KEISHI NAKAMURA ${ }^{1}$, HIRONORI HAYASHI ${ }^{1}$, \\ KATSUNOBU OYAMA ${ }^{1}$, MASAFUMI INOKUCHI ${ }^{1}$, HISATOSHI NAKAGAWARA ${ }^{1}$, HIDETO FUJTA ${ }^{1}$, \\ HIROYUKI TAKAMURA $^{1}$, ITASU NINOMIYA ${ }^{1}$, SACHIO FUSHIDA ${ }^{1}$, TAKASHI TANI ${ }^{1}$, \\ TAKASHI FUJIMURA ${ }^{1}$, HIROKO IKEDA $^{2}$ and SEIKO KITAMURA ${ }^{2}$
}

${ }^{1}$ Department of Gastroenterologic Surgery, Division of Cancer Medicine, Graduate School of Medicine Science, Kanazawa University; ${ }^{2}$ Division of Pathology, Kanazawa University Hospital, Kanazawa, Japan

Received September 25, 2011; Accepted November 25, 2011

DOI: $10.3892 / \mathrm{etm} .2012 .482$

\begin{abstract}
Results of surgery alone for pancreatic cancer are disappointing. We retrospectively evaluated the efficacy and tolerability of neoadjuvant chemotherapy (NAC) with gemcitabine and oral S-1 in patients with potentially resectable pancreatic cancer. A total of 34 patients with pancreatic ductal adenocarcinoma, radiologically diagnosed preoperatively as having potentially resectable tumors, underwent pancreatic resection with lymphadenectomy at Kanazawa University Hospital. NAC was administered to 13 patients (NAC group). The remaining 21 patients were surgically treated without preoperative chemotherapy (control group). Surgical results were compared between these two groups, with follow-up for at least 24 months. No statistically significant differences were found in the clinicopathological background data (tumor location, age, gender, lymph node metastases, tumor stage and tumor size) between the NAC and control groups. Following preoperative chemotherapy, no patients were judged to be unable to undergo laparotomy, i.e., neither distant metastasis nor tumor progression was observed. Radiologically, all 13 NAC group patients had stable disease, whereas, histopathologically, all tumor specimens showed evidence of tumor cells. The treatment effect was judged by Evans grading to be grade IIa in 11 patients and grade IIb in 2 patients. Toxicity was evaluated in 11 patients. Grade III side effects were regarded as hematological toxicity, i.e., leucopenia (7.7\%) and thrombocytopenia (15.4\%). Moreover, the incidence of perioperative
\end{abstract}

Correspondence to: $\mathrm{Dr}$ Hidehiro Tajima, Department of Gastroenterologic Surgery, Division of Cancer Medicine, Graduate School of Medicine Science, Kanazawa University, 13-1 Takaramachi, Kanazawa 920-8641, Japan

E-mail: hidetaji@staff.kanazawa-u.ac.jp

Key words: pancreatic cancer, gemcitabine, S-1, neoadjuvant chemotherapy complications did not differ significantly between the NAC and control groups. The one- and three-year overall survival rates of the NAC group with pancreatic head cancer were 88.9 and $55.6 \%$, respectively, superior to 88.9 and $29.6 \%$ in the control group $(\mathrm{p}=0.055)$. Therefore, NAC with gemcitabine and $\mathrm{S}-1$ is well tolerated and potentially effective against pancreatic head cancer. A phase I study of NAC with gemcitabine and S-1 is under way in patients with resectable pancreatic cancer.

\section{Introduction}

Pancreatic carcinoma causes more than 20,000 deaths every year in Japan and overall five-year survival is less than 5\% $(1,2)$. For patients with localized disease, radical surgery may be of long-term benefit. Therefore, we usually perform radical pancreatic resection, comprising wide lymph node dissection and complete removal of the extrapancreatic nerve plexus of the superior mesenteric artery or celiac axis for patients with carcinoma of the pancreas, to improve outcomes (3-5). However, even in patients who undergo resection, five-year survival remains only $7-24 \%$ and median survival is only approximately one year in most series, indicating that surgery alone is usually inadequate. These disappointing results are likely attributable to early vascular dissemination; i.e. subclinical metastases are present at the time of diagnosis in most patients (6). This hypothesis underpins the investigation of adjuvant chemotherapy after surgery. Oettle et al reported that adjuvant chemotherapy with gemcitabine produced a statistically significant improvement in overall survival (OS) (7).

A major drawback of adjuvant therapy for pancreatic cancer is the marked and consistent failure of $20-30 \%$ of patients to receive the designated therapy as a result of post-operative complications, delayed surgical recovery, patient refusal, comorbidity, or early disease recurrence (8-10). These challenges can be overcome in certain cases by administering preoperative therapy, in order that more patients receive potentially beneficial adjuvant treatment. Other theoretical advantages of this approach include the early treatment of micrometastases, delaying surgery and thereby sparing 
those who already have occult metastases the morbidity and mortality of major surgery if disseminated disease becomes apparent at the time of reassessment; reduced risk of intraoperative tumor seeding; better treatment tolerance than with postoperative therapy; and reduced overall treatment time.

Potential disadvantages of preoperative therapy include the requirement for biliary decompression before chemotherapy with the potential for complications associated with biliary stents; delayed surgery allowing progression to a non-resectable stage in patients whose disease did not respond to therapy; lack of preoperative tissue diagnosis (risk of seeding if a preoperative biopsy is performed); and more postoperative complications.

Gemcitabine is a deoxycytidine analogue that competes for incorporation into DNA, thereby inhibiting its synthesis. Gemcitabine is currently the standard treatment for advanced pancreatic cancer on the basis of a randomized study comparing gemcitabine with 5-fluorouracil (5-FU) in 126 patients in which a small but clinically important survival advantage and greater clinical benefit favoring gemcitabine were observed.

S-1 is an oral fluorinated pyrimidine developed by Taiho Pharmaceutical Co., Ltd. (Tokyo, Japan). This agent contains tegafur (FT), 5-chloro-2,4-dihydroxypyridine (CDHP), and potassium oxonate (Oxo) at a molar ratio (FT:CDHP:Oxo) of 1:0.4:1, based on a biochemical modulation of 5-FU (11). FT, a prodrug of 5-FU, is gradually converted into 5-FU and is rapidly catabolized by dihydropyrimidine dehydrogenase (DPD) in the liver. 5-Chloro-2,4-dihydroxypyridine is a competitive inhibitor of 5-FU catabolism, being about 180 times more potent than uracil in inhibiting DPD (12). When FT is combined with CDHP, the resulting high 5-FU levels are maintained in both plasma and the tumor. In addition, it has been suggested that CDHP has the potential to enhance the antitumor activity of 5-FU against subcutaneous tumors in nude mice, using human pancreatic carcinoma cells with highly malignant DPD activity (13). Oxo inhibits the enzyme orotate phosphoribosyltransferase, the major enzyme responsible for 5-FU activation in colon cancer (14). Oxo preferentially localizes in the gut rather than in the tumor and appears to have a biochemical effect on orotate phosphoribosyltransferase, thereby selectively inhibiting the formation of 5-FU nucleotides in the gut and theoretically reducing gastrointestinal side effects (15). Oral administration of S-1 is more convenient and simulates the effect of continuous infusion of 5-FU. The safety and usefulness of combination chemotherapy with gemcitabine and S-1 for advanced pancreatic cancer were previously reported (16-18) and a phase III trial was conducted in Japan.

The usefulness of preoperative (neoadjuvant) gemcitabinebased chemotherapy or chemoradiotherapy for prolonging survival in patients with resectable pancreatic cancer was also previously reported (19-22). As yet, the combination regimen of gemcitabine and S-1 for patients with preoperatively resectable pancreatic cancer has not been investigated. Therefore, we conducted a pilot study of neoadjuvant chemotherapy (NAC) with a combination of gemcitabine plus S-1 for patients with resectable pancreatic cancer.

\section{Materials and methods}

Patient selection. Between January 2006 and June 2009, 35 patients who had radiologically diagnosed pancreatic cancer regarded as potentially resectable underwent pancreatic resection with lymphadenectomy at Kanazawa University Hospital. A total of 34 of the 35 resected tissue specimens were pathologically proven to be ductal adenocarcinoma of the pancreas. NAC was administered to 13 (NAC group) of the 34 patients. The other 21 patients were surgically treated without preoperative chemotherapy (control group). The study was non-randomized.

Written informed consent was obtained from each patient prior to enrollment, and the local ethics committee approved the study.

Eligibility criteria included being 20-79 years of age, Eastern Cooperative Oncology Group (ECOG) performance status of one or less (ambulatory and capable of self-care), adequate renal function (normal serum creatinine and blood urea nitrogen levels), adequate liver function (total bilirubin level $<2.5$ times upper normal limit (UNL) or $<3$ times UNL after biliary drainage if the patient had jaundice and serum transaminases (GOT, GPT) levels $<2.5$ or 3 times UNL), bone marrow reserve (white blood cell count between 4000 and $12,000 \mathrm{~mm}^{3}$, neutrophil count $>2000 \mathrm{~mm}^{3}$, platelet count $>100,000 \mathrm{~mm}^{3}$, hemoglobin $>9.5 \mathrm{~g} / \mathrm{dl}$ ), and adequate pulmonary function $\left(\mathrm{PaO}_{2}>65 \mathrm{mmHg}\right)$. Any prior cancer treatments (tumor resection, chemotherapy, immunotherapy, or radiotherapy) had to have been discontinued at least four weeks prior to study entry.

The exclusion criteria included pulmonary fibrosis or interstitial pneumonia, marked pleural or pericardial effusion or marked peripheral edema, most forms of heart disease, difficult to control diabetes mellitus, active infection, pregnancy or lactation, being a woman of childbearing age unless using effective contraception, severe drug hypersensitivity, appearance of distant metastases during preoperative chemotherapy, severe neurological impairment or mental disorder, active concomitant malignancy, and other serious medical conditions.

Treatment protocol. The laboratory tests required to assess eligibility had to be completed within seven days prior to the start of treatment; S-1 was administered orally after a meal for 14 consecutive days (from the evening of day 1 to the morning of day 15), followed by a one-week break. Each capsule of $\mathrm{S}-1$ contained 20 or $25 \mathrm{mg}$ of FT. Individual doses were rounded down to the nearest pill size less than the calculated dose, given the available formulation. Gemcitabine was administered as a 30-min intravenous infusion on days 8 and 15 of each cycle. The cycle was repeated twice. This schedule was based on an in vitro study, which showed maximum synergy when fluoropyrimidine precedes exposure to gemcitabine (23). The planned drug doses in this study were as follows: $\mathrm{S}-130 \mathrm{mg} / \mathrm{m}^{2}$ day and gemcitabine $800 \mathrm{mg} / \mathrm{m}^{2}$ (Fig. 1). The two groups received pancreatectomy with lymphadenectomy. In cases with suspected tumor infiltration into the portal vein, resection of the vein with reconstruction was performed. In the NAC group, the operation was performed more than two weeks after chemotherapy.

Assessment of efficacy. Tumor responses were evaluated according to Response Evaluation Criteria In Solid Tumors (RECIST). A complete response (CR) was defined as the disappearance of all clinical evidence of the measurable tumor. 


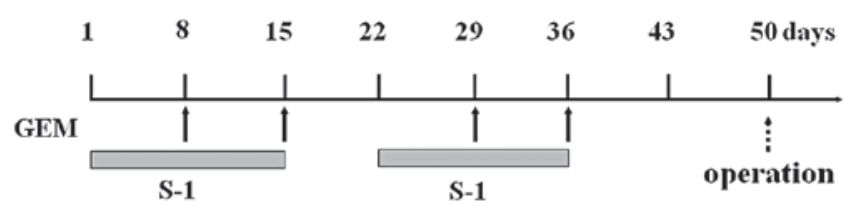

\begin{tabular}{|l|c|c|} 
GEM $: 800 \mathrm{mg} / \mathrm{m}^{2}$ & Body Surface Area & $\mathrm{S}-1$ \\
\cline { 2 - 3 } $\mathrm{S}-1: 30 \mathrm{mg} / \mathrm{m}^{2}$ & $<1.34 \mathrm{~m}^{2}$ & $30 \mathrm{mg} /$ day \\
\hline & $1.34 \mathrm{~m}^{2}$ to $1.5 \mathrm{~m}^{2}$ & $40 \mathrm{mg} /$ day \\
\hline$>1.5 \mathrm{~m}^{2}$ & $50 \mathrm{mg} /$ day \\
\hline
\end{tabular}

Figure 1. Treatment protocol of neoadjuvant chemotherapy with gemcitabine and S-1. S-1 $30 \mathrm{mg} / \mathrm{m}^{2} /$ day was administered orally for 14 consecutive days, and gemcitabine $800 \mathrm{mg} / \mathrm{m}^{2}$ was administered on days 8 and 15 . In the NAC group, the operation was performed more than two weeks after two cycles of chemotherapy.

A partial response (PR) was defined as a $\geq 30 \%$ reduction in the sum of the products of two perpendicular diameters of all measurable lesions compared to the base-line with no evidence of new lesions. Stable disease (SD) was defined as a $<30 \%$ reduction or $<20 \%$ increase in the sum of the products of two perpendicular diameters of all measurable lesions compared to the base-line, with no evidence of new lesions. Progressive disease (PD) was defined as an increase of $\geq 20 \%$ in the sum of the products of two perpendicular diameters of all measurable lesions compared to the base-line, the appearance of any new lesion, or deterioration in clinical status consistent with disease progression. To assess objective responses, patients were evaluated following two cycles of chemotherapy, i.e., prior to surgery.

Pathological diagnosis. Surgically resected specimens were immediately fixed in a $10 \%$ neutral-buffered formaldehyde solution. After the specimens had been cut horizontally into 5-mm tissue blocks and corresponding computerized tomographic (CT) images taken (24), they were dehydrated and embedded in paraffin. Sections $(5-\mu \mathrm{m})$ were cut and stained with hematoxylin and eosin. Each section was carefully examined by light microscopy. The tumors were evaluated according to the General Rules for the Clinical and Pathological Study of Pancreatic Cancer proposed by the Japanese Pancreatic Cancer Group. The grading system of Evans et al was used to assess the pathological effects of preoperative chemotherapy (25). The degrees of cytological change and tumor destruction were graded on a scale of I-IV, as follows: grade I, characteristic cytologic changes of malignancy are present, but little $(<10 \%)$ or no tumor cell destruction is evident; grade IIa, destruction of $10-50 \%$ of tumor cells; grade IIb, destruction of $51-90 \%$ of tumor cells; grade III, few $(<10 \%)$ viableappearing tumor cells are present; grade IIIM, sizable pools of mucin are present; grade IV, no viable tumor cells are present; grade IVM, acellular pools of mucin are present.

Follow-up. Adjuvant chemotherapy with bi-weekly gemcitabine was administered following surgery, if possible. Patients were evaluated by physical examination and laboratory data bi-weekly and by CT every three months.
Table I. Patient characteristics.

\begin{tabular}{lccc}
\hline Characteristics & NAC group & Control group & p-value \\
\hline $\begin{array}{l}\text { Patients (n) } \\
\text { Gender (n) }\end{array}$ & 13 & 21 & \\
$\quad$ Male & & & \\
$\quad$ Female & 6 & 14 & NS \\
$\begin{array}{l}\text { Age (years) } \\
\quad \text { Median }\end{array}$ & 62.6 & 66.0 & NS \\
$\quad$ Range & $51-77$ & $52-80$ & \\
$\begin{array}{l}\text { Location (n) } \\
\text { Head of pancreas }\end{array}$ & 9 & 11 & NS \\
Pancreas body and tail & 3 & 10 & \\
\hline
\end{tabular}

NS, not significant.

Statistical analysis. Categorical variables were compared using the Chi-square test. The OS and disease-free survival (DFS) rates were calculated from the start of the study or study treatment until death or the final date of follow-up and determined by the Kaplan-Meier method. Data for surviving patients at the time of the study report were censored. The log-rank test was applied for comparison of survival rates between groups. Results were considered significant at $\mathrm{p}<0.05$.

\section{Results}

Clinical data of patients. From 2006 to June 2009, 13 patients (7 males and 6 females) diagnosed as having resectable pancreatic ductal adenocarcinoma were treated with NAC using S-1 and gemcitabine. Their mean age was 62.6 years (range, 51-77). There were 21 patients ( 14 males and 7 females) in the control group, with a mean age of 66.0 years (range, 52-80). No differences were found between the groups in terms of age, gender, status of lymph node metastases, tumor stage or tumor size (Table I). Following chemotherapy, no patients were judged to be incapable of undergoing laparotomy. Neither distant metastasis nor tumor progression was observed.

In the NAC group, comprising 13 ductal adenocarcinoma patients, nine with pancreatic head cancer were treated by pancreaticoduodenectomy with portal resection and five with pancreatic body-to-tail cancer underwent distal pancreatectomy. In the control group, comprising 21 ductal adenocarcinoma patients, 11 patients with pancreatic head cancer were treated by pancreaticoduodenectomy with portal resection, and 10 with pancreatic body-to-tail cancer underwent distal pancreatectomy. Of the 13 patients (69.2\%), 9 completed both courses of preoperative chemotherapy.

Response to chemotherapy. The 13 NAC patients exhibited $\mathrm{SD}$. The radiological findings showed the average rate of tumor shrinkage was $8.1 \%$ (-5.7 to $25.8 \%)$. None of the patients showed an increase in tumor size during preoperative chemotherapy, but in half of the patients the tumor size was unchanged. In the NAC group, the tumor specimens exhibited histopathological evidence of tumor cell injury, although none of the patients 
Table II. Histopathological characteristics.

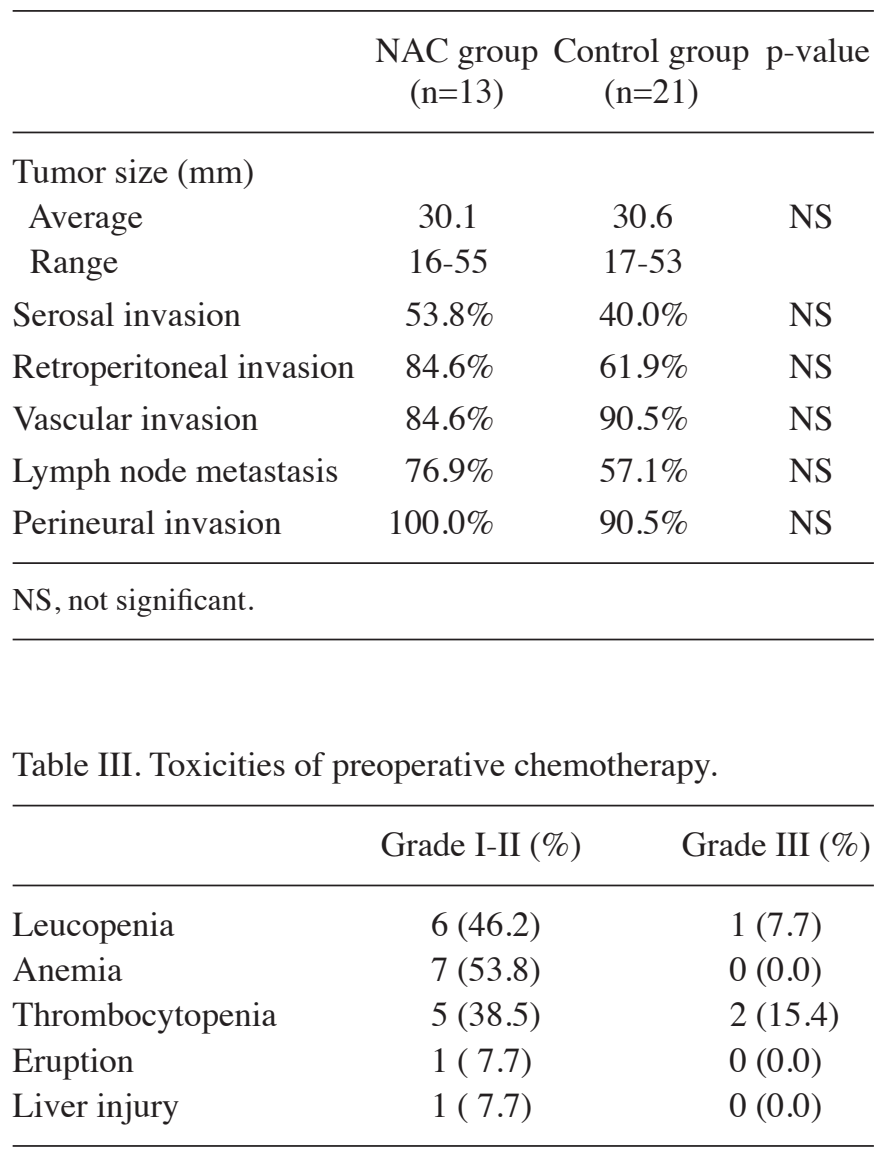

exhibited a pathological CR. The treatment effect, as judged by the Evans grading system, was grade IIa in 11 patients and grade IIb in two.

Surgical results. Intraoperative blood loss during pancreatic cancer resection was $487.3 \pm 326.8 \mathrm{ml}$ in the NAC group and $487.6 \pm 390.0 \mathrm{ml}$ in the control group. No significant difference was found between the two groups in terms of tumor location (Table II). The overall postoperative mortality rate was $0 \%$. In the NAC group, eight patients (61.5\%) had complications. One case each developed pancreatic fistula, delayed gastric empty (DGE) and intra-abdominal infections, which were treated conservatively. In the control group, 12 patients $(57.1 \%)$ suffered complications. There were four intra-abdominal infections, five DGE, two pancreatic fistulas, and one case each of ileus and wound infection, both of which were treated conservatively. However, one case with a pancreatic fistula complicated by hepatic arterial bleeding required interventional radiology.

Efficacy. Median resected tumor specimen size was $30.1 \mathrm{~mm}$ in the NAC group, with no patients demonstrating a pathological CR (Table II). Tumor size in the control group was $30.6 \mathrm{~mm}$, not significantly larger than in the NAC group. The NAC and control groups did not differ significantly in the frequency of lymph node metastasis or infiltration of vessels, nerve plexuses and the retroperitoneum. Moreover, the frequency of pathologically curative resection (R0) in the NAC group was not significantly higher than that in the control group (R0/1/2\%, 84.6/15.4/0.0 versus 85.7/14.3/0.0).
Table IV. Patterns of recurrence in resected patients.

\begin{tabular}{lccc}
\hline & $\begin{array}{c}\text { NAC group } \\
(\mathrm{n}=7, \%)\end{array}$ & $\begin{array}{c}\text { Control } \\
(\mathrm{n}=13, \%)\end{array}$ & p-value \\
\hline Local & $1(14.3)$ & $1(7.7)$ & $\mathrm{NS}$ \\
Liver & $4(57.1)$ & $10(76.9)$ & $\mathrm{NS}$ \\
Peritoneum & $1(14.3)$ & $4(30.8)$ & $\mathrm{NS}$ \\
Lung & $3(42.9)$ & $0(0.0)$ & $\mathrm{NS}$ \\
Lymph node & $1(14.3)$ & $1(7.7)$ & $\mathrm{NS}$ \\
\hline
\end{tabular}

NS, not significant.

Toxicity. Eleven of the 13 NAC patients were evaluated for toxicity. The most common side effects are shown in Table III. A substantial percentage of NAC patients experienced grades I-III hematological toxicities, i.e., leucopenia (grades I-II, 46.2\%; grade III, 7.7\%), anemia (grades I-II, 53.8\%), and thrombocytopenia (grades I-II, 38.5\%; grade III, 15.4\%). Other side effects were liver injury (grade I, 8.3\%) and eruption (grade I, 8.3\%).

OS and DFS rates. Of the 34 patients, 17 (50.0\%) patients were followed up with adjuvant chemotherapy with bi-weekly gemcitabine administration following surgery. The median follow-up time after the operation was 30.0 months (range, 24-48) for all patients. Although the one- and three-year OS rates of pancreatic head cancer patients in the NAC group were 88.9 and $55.6 \%$, superior to the 88.9 and $29.6 \%$ in the control group $(\mathrm{p}=0.055)$, the differences did not reach statistical significance (Fig. 2A). OS rates for pancreatic body and tail cancer patients did not differ significantly between the two groups (Fig. 2B).

No significant differences were observed in the DFS rates of pancreatic head cancer versus pancreatic body-to-tail cancer patients, and between the NAC and control groups (Fig. 3A and B). Sites of recurrence are shown in Table IV. There were significant differences between the two groups. However, the number of liver metastases tended to be lower in the NAC $(1.7 \pm 0.6)$ than in the control $(5.8 \pm 4.3)$ group $(\mathrm{p}=0.065)$.

\section{Discussion}

Curative surgical resection is the only means of curing pancreatic cancer. However, the majority of pancreatic cancer resections are reportedly R1 (26) and, even after undergoing curative resection, patients with pancreatic cancer face a $50-80 \%$ local recurrence rate and a $25-50 \%$ chance of developing distant metastases (6). We reported that for patients with localized pancreatic cancer, radical pancreatic resection, consisting of wide lymph node dissection and complete removal of the extrapancreatic nerve plexus of the superior mesenteric artery or celiac axis, improves outcomes (3-5). However, the long-term results are not satisfactory due to the high frequency of distant metastasis. Given the unsatisfactory outcomes obtained to date, adjuvant chemotherapy is required. In particular, pancreatic head cancer, partly due to surgical stress, requires a long postoperative recovery period before 
A

Pancreatic head cancer

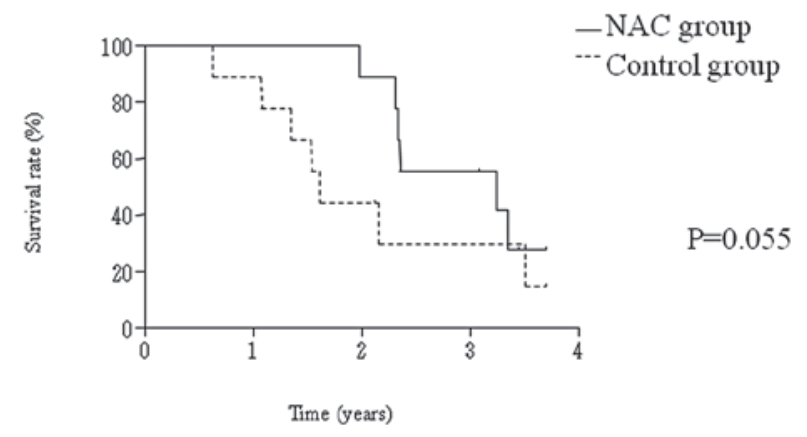

B

Pancreatic body and tail cancer

Figure 2. Overall survival (OS) curve for (A) pancreatic head cancer and (B) pancreatic body-tail cancer.

A

Pancreatic head cancer

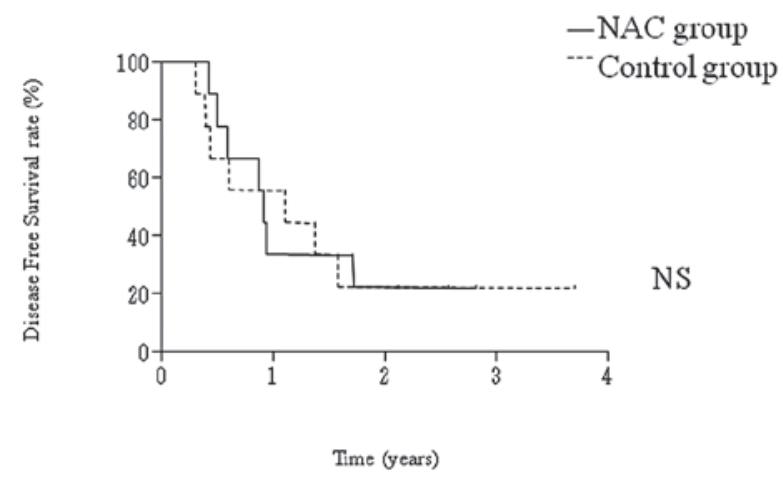

B

Pancreatic body and tail cancer

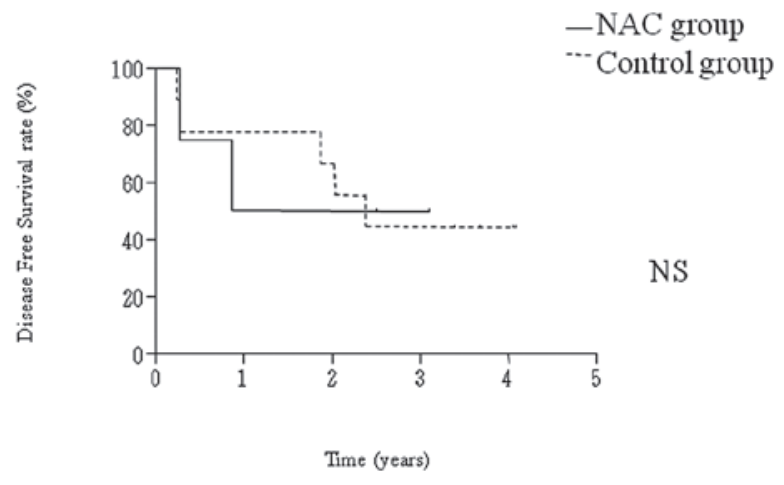

Figure 3. Disease free survival (DFS) curve for (A) pancreatic head cancer and (B) pancreatic body-tail cancer.

chemotherapy is administered. Preoperative chemotherapy is expected to reduce the risk of distant metastasis.

Nakamura et al conducted a phase II clinical trial of S-1 combined with gemcitabine for metastatic pancreatic cancer (16). In that trial, S-1 was administered for 14 consecutive days prior to gemcitabine. Moreover, Nakahira et al reported that pretreatment with S-1 enhances gemcitabine effects in pancreatic cancer xenografts (27). The mechanism of these enhanced effects is considered to be 5-FU upregulation of the major mediator of cell uptake of gemcitabine, the human equilibrative nucleoside transporter 1 (hENT1). In this study, we adopted the regimen of Nakamura et al (16). However, due to the high incidence of side effects with this preoperative chemotherapy, the S-1 dose was reduced. In other words, S-1 was used only as a biochemical modulator of gemcitabine.

Identifying an objective response following chemotherapy for localized pancreatic cancer is extremely difficult. Pancreatic cancer can be responsive to treatment even if the histological response to chemotherapy, the radiological findings, and tumor size were only minimally altered. Therefore, the viability of tumors, as measured by positron emission tomography-CT, has been used for response evaluation.

The effectiveness of preoperative chemoradiotherapy has previously been reported $(25,28,29)$. Chemoradiotherapy is superior for local control of pancreatic cancer, but one report described a higher rate of distant metastases compared to chemotherapy alone (30). Based on these factors, we performed radical resection for local control of pancreatic cancer, rather than administering radiation therapy preoperatively.

In the nine NAC-group patients with pancreatic head cancer, OS was prolonged compared to the control group. Although the difference did not reach statistical significance, there were no deaths within a two-year postoperative period in the NAC-group patients with pancreatic head cancer. Notably, pancreaticoduodenectomy for pancreatic head cancer is highly invasive. Therefore, a few months are required to recover from surgery, before adjuvant chemotherapy can be administered. Furthermore, chemotherapy dose reductions are required for patients who are not able to tolerate full doses of anticancer drugs. Preoperative chemotherapy may be advantageous in allowing time for postoperative chemotherapy. In addition, fewer liver metastases were evident in the NAC compared to the control group. These factors may have contributed to prolonged DFS.

Of the 13 NAC patients, $11(84.6 \%)$ patients were evaluated for toxicity. No severe toxicities were observed. In addition, incidences of perioperative complications did not differ significantly between the two groups. 
In conclusion, NAC with gemcitabine and S-1 is well tolerated and may be effective, particularly against pancreatic head cancer. However, the appropriate doses of anticancer drugs should be deteremined in a future study. A phase I study of NAC with gemcitabine and S-1 is currently under way in patients with resectable pancreatic cancer.

\section{References}

1. Ministry of Health, Labour and Welfare: The Dynamic Statistics of the Population in 2005. http//www.mhlw.go.jp/toukei/ saikin/hw/jinkou/ kakutei05/hyo7/html (11 June 2009, data last accepted).

2. Ishii H, Furuse J, Boku N, et al: Phase II study of gemcitabine chemotherapy alone for locally advanced pancreatic carcinoma: JCOG0506. Jpn J Clin Oncol doi:10.1093/jjco/hyq011, 2010.

3. Nagakawa T, Kurachi M, Konishi K, et al: Translateral retroperitoneal approach in radical surgery for pancreatic carcinoma. Jpn J Surg 12: 229-233, 1982 .

4. Nagakawa T, Nagamori M, Futakami F, et al: Result of extensive surgery for pancreatic carcinoma. Cancer 77: 640-645, 1996.

5. Miwa K, Ohta T, Shimizu K, et al: Augmented regional pancreatoduodenectomy for pancreas head cancer: combined resection of pancreas head and superior mesenteric artery and vein. American College of Surgeons 90th Annual Clinical Congress, Chicago:Isis 190, 2004.

6. Evans DB, Abbruzzese JL and Willett CG: Cancer of the pancreas. In: Cancer: Principles and Practice of Oncology. 6th edition. De Vita, Hellman S and Rosenberg SA (eds). Lippincott, Williams and Wilkins, Philadelphia, pp1126-1161, 2001.

7. Oettle H, Post S, Neuhaus P, et al: Adjuvant chemotherapy with gemcitabine vs observation in patients undergoing curativeintent resection of pancreatic cancer: a randomized controlled trial. JAMA 297: 267-277, 2007.

8. Klinlenbijl JH, Jeekel J, Sahmoud T, et al: Adjuvant radiotherapy and 5-fluorouracil after curative resection of cancer of the pancreas and periampullary region: phase III trial of the EORTC Gastrointestinal Tract Cancer Cooperative Group. Ann Surg 230: 776-782, 1999

9. Spitz FR, Abbruzzese JL, Lee JE, et al: Preoperative and postoperative chemoradiation strategies in patients treated with pancreaticoduodenectomy for adenocarcinoma of the pancreas. J Clin Oncol 15: 928-937, 1997.

10. Yeo CJ, Abrams RA, Grochow LB, et al: Pancreaticoduodenectomy for pancreatic adenocarcinoma: postoperative adjuvant chemoradiation improves survival. A prospective, single-institution experience. Ann Surg 225: 621-633, 1997.

11. Shirasaka T, Shimamoto Y, Ohshimo H, et al: Development of a novel form of an oral 5-fluorouracil derivative (S-1) directed to the potentiation of the tumor selective cytotoxicity of 5-fluorouracil by two biochemical modulators. Anticancer Drugs 7: 548-557, 1996.

12. Tatsumi K, Fukushima M, Shirasaki T and Fujii S: Inhibitory effects of pyrimidine, barbituric acid and pyrimidine derivatives on 5-fluorouracil degradation in rat liver extracts. Jpn J Cancer Res 78: 748-755, 1987.

13. Takechi T, Fujioka A, Matssushima E, et al: Enhancement of the antitumor activity of 5-fluorouracil (5-FU) by inhibiting dihydropyrimidine dehydrogenase activity (DPD) using 5-chloro-2,4-dihydroxypyridine (CDHP) in human tumor cells. Eur J Cancer 38: 1271-1277, 2002.
14. Peters GJ, van Groeningen CJ, Laurensse EJ and Pinedo HM: A compression of 5-fluorouracil metabolism in human colorectal cancer and colon mucosa. Cancer 68: 1903-1909, 1991.

15. Takechi T, Nakano K, Uchida J, et al: Antitumor activity and low intestinal toxicity of $\mathrm{S}-1$, a new formulation of oral tegafur. in experimental tumor models in rats. Cancer Chemother Pharmacol 39: 205-211, 1997.

16. Nakamura K, Yamaguchi T, Ishihara T, et al: Phase I trial of oral $\mathrm{S}-1$ combined with gemcitabine in metastatic pancreatic cancer. Br J Cancer 92: 2134-2139, 2005.

17. Ueno H, Okusaka T, Ikeda M, et al: A phase I study of combination chemotherapy with gemcitabine and oral S-1 for advanced pancreatic cancer. Oncology 69: 421-427, 2005.

18. Nakamura K, Yamaguchi T, Ishihara T, et al: Phase II trial of oral S-1 combined with gemcitabine in metastatic pancreatic cancer. Br J Cancer: 1-5, 2006.

19. Vento P, Mustonen H, Joensuu T, et al: Impact of preoperative chemoradiotherapy on survival in patients with resectable pancreatic cancer. World J Gastroenterol 21: 2945-2951, 2007.

20. Palmer DH, Stocken DD, Hewitt H, et al: A randomized phase 2 trial of neo-adjuvant chemotherapy in resectable pancreatic cancer: gemcitabine alone versus gemcitabine combined with cisplatin. Ann Surg Oncol 14: 2088-2096, 2007.

21. Sato N, Kurashima K, Nagai H, et al: The effect of adjuvant and neoadjuvant chemo (radio) therapy on survival in 1,679 resected pancreatic carcinoma cases in Japan: report of the national survey in the 34th annual meeting of Japanese Society of Pancreatic Surgery. J Hepatobiliary Pancreat Surg 16: 485-492, 2009.

22. Satoi S, Yanagimoto $\mathrm{H}$, Toyokawa $\mathrm{H}$, et al: Surgical result after preoperative chemoradiation therapy for patients with pancreatic cancer. Pancreas 38: 282-288, 2008.

23. Rauchwerger DR, Firby PS, Hedley DW and Moore MJ: Equilibrative-sensitive nucleoside transporter and its role in gemcitabine sensitivity. Cancer Res 60: 6075-6079, 2000.

24. Makino I, Kitagawa H, Ohta T, et al: Nerve plexus invasion in pancreatic cancer. Spread patterns on histopathologic and embryological analysis. Pancreas 37: 358-365, 2008.

25. Evans DB, Rich TA, Byrd DR, et al: Preoperative chemoradiation and pancreaticoduodenectomy for adenocarcinoma of the pancreas. Arch Surg 127: 1335-1339, 1992.

26. Esposito I, Kleeff J, Bergmann F, et al: Most pancreatic cancer resections are R1 resections. Ann Surg Oncol 15: 1651-1660, 2008.

27. Nakahira S, Nakamori S, Tsujie M, et al: Pretreatment with S-1, an oral derivative of 5-fluorouracil, enhances gemcitabine effect in pancreatic cancer xenografts. Anticancer Res 28: 179-186, 2008.

28. Takai S, Satoi S, Yanagimoto H, et al: Neoadjuvant chemoradiation in patients with potentially resectable pancreatic cancer. Pancreas 36: 26-32, 2008.

29. Golcher H, Brunner T, Grabenbauer G, et al: Preoperative chemoradiation in adenocarcinoma of the pancreas. A single centre experience advocating a new treatment strategy. EJSO 34: 756-764, 2008.

30. Van Laethem JL, Hammel P, Mornex F, et al: Adjuvant gemcitabine alone versus gemcitabine-based chemoradiotherapy after curative resection for pancreatic cancer: A randomized EORTC-40013-22012 /FFCD-9203/GERCOR phase II study. J Clin Oncol 28: 4450-4456, 2010. 